\title{
Biodigestor con variables monitoreadas
}

\section{Biodigestor with monitored variables}

ORTEGA-BUCIO, Lydia Gabriela†**, HERNÁNDEZ-HERNÁNDEZ, Fabiola, ZEA-PÉREZ, José Marcos y BECERRA-CHAVEZ, Adela

Universidad Politécnica de Querétaro, UPQ

ID $1^{\text {er }}$ Autor: Lydia Gabriela, Ortega-Bucio / ORC ID: 0000-0001-9004-4397, CVU CONACYT ID: 58672

ID $1^{\text {er }}$ Coautor: Fabiola, Hernández-Hernández / ORC ID: 0000-0002-6106-5460, Researcher ID Thomson: X-3885-2018, CVU CONACYT ID: 240104

ID $2^{\text {do }}$ Coautor: José Marcos, Zea-Pérez / ORC ID: 0000-0002-7220-1923, CVU CONACYT ID: 269210

ID $3^{\text {er }}$ Coautor: Adela, Becerra-Chavez / ORC ID: 0000-0001-8752-391X, Researcher ID Thomson: X-3894-2018, CVU CONACYT ID: 953424

DOI: $10.35429 / J C P E .2019 .18 .6 .37 .47$

Recibido 09 Febrero, 2019; Aceptado 28 Marzo, 2019

\section{Resumen}

El proyecto consiste en el diseño y construcción de un Biodigestor que permita la generación de gas metano para calentamiento y cocción de alimentos en estufas domésticas. El Biodigestor tendrá como subproducto Biol, proveniente del lixiviado de la materia orgánica, este subproducto puede ser utilizado como fertilizante en jardines o cultivos domésticos. Las variables monitoreadas del Biodigestor utilizan una tarjeta de adquisición de datos para obtener el nivel de $\mathrm{Ph}$ en el biol, la temperatura, humedad y presión durante el proceso de generación de metano, así como la cantidad y calidad de gas metano producido. Este proyecto se ha desarrollado en varias etapas: diseño, pruebas en prototipo a escala, conexión de sensores y pruebas que permiten definir datos de referencia y datos durante la producción de metano. Se ha logrado un diseño que permite la producción simultánea en tres biodigestores, esta propuesta permitirá que a través de un protocolo específico, se podrá mantener la continuidad en la obtención del gas teniendo la posibilidad de vaciar, limpiar y sustituir la materia orgánica sin parar el proceso de producción de metano.

Biodigestor, Monitorear, Metano

\begin{abstract}
The project consists of the design and construction of a biodigester that allows the generation of methane gas for heating and cooking food in domestic stoves. The Biodigestor will have as byproduct Biol, from leached organic matter, this byproduct can be used as fertilizer in gardens or domestic crops. The monitored variables of the Biodigestor use a data acquisition card to obtain the level of $\mathrm{Ph}$, biol, temperature, humidity and pressure during the methane generation process, as well as the quantity and quality of methane gas produced. This project has been developed in several stages, design, prototype scale tests, connection of sensors and tests that allow to define reference data and data during methane production. A design has been achieved that allows simultaneous production in three biodigestors, this proposal will allow through a specific protocol, the continuity in obtaining the gas can be maintained with the possibility of emptying, cleaning and replacing organic matter without stopping the methane production process.
\end{abstract}

Biodigestor, Monitoring, Methane

Citación: ORTEGA-BUCIO, Lydia Gabriela, HERNÁNDEZ-HERNÁNDEZ, Fabiola, ZEA-PÉREZ, José Marcos y BECERRA-CHAVEZ, Adela. Biodigestor con variables monitoreadas. Revista de Energía Química y Física. 2019. 6-18: 3747

\footnotetext{
* Correspondencia al Autor (Correo electrónico: lydia.ortega @upq.mx)

$\dagger$ Investigador contribuyendo como primer Autor
} 


\section{Introducción}

Las energías alternativas constituyen una opción importante para sustentar los requerimientos domésticos del combustible fósil y procesos productivos actuales, disminuyendo costos $\mathrm{y}$ mejorando las condiciones ambientales de nuestro país. Dentro de las energías alternativas, en este proyecto estaremos abordando el sistema que conforman los Biodigestores, los que permiten la generación de gas metano a partir de desechos orgánicos, logrando la obtención de energía limpia a bajo costo a partir de una fuente renovable.

La construcción de un Biodigestor con variables monitoreadas permite la supervisión del proceso y el control del producto generado, el cual requiere de características específicas como son:

\section{- $\quad$ La temperatura y el $\mathrm{pH}$ \\ - Concentración de sólidos y sustancias inhibidoras de la reacción biológica \\ - $\quad$ Adición de sustancias para el equilibrio de niveles ambientales y biológicos adecuados}

El desarrollo de este proyecto se ha implementado con una metodología científica tradicional que consiste en:

- Establecer propuestas y analizar la viabilidad de estas en las condiciones económicas y técnicas que requiere el proyecto y de las que se disponen con recursos materiales y humanos.

- Diseñar el prototipo definitivo, lo que permitirá identificar de manera específica los requerimientos técnicos.

- Integrar los elementos mecánicos, electrónicos y químicos para realizar las pruebas de funcionalidad.

- Verificar la funcionalidad del prototipo y la calidad del producto obtenido, lo que permite que se establezcan las pruebas necesarias que se requieran para verificar y alcanzar los objetivos.

Las actividades realizadas se han desarrollado en dos etapas principales:

Etapa 1. Construcción de prototipo a escala que permita realizar pruebas de sensores.

Etapa 2. Establecer diseño definitivo para el Biodigestor controlado

La hipótesis planteada establecida en el proyecto queda de la siguiente manera:

Se pueden controlar las variables de un Biodigestor para eficientar la producción de gas metano y biol.

\section{Sustento Teórico}

Realizar el monitoreo de variables de un Biodigestor, requiere de comprender el proceso del sistema de descomposición de la materia orgánica dentro del sistema conocido como Biodigestor, para ello, en este apartado se presenta el fundamento teórico del biodigestor, sus productos y su instrumentación para el monitoreo de las variables requeridas.

\section{Biodigestores clasificados de acuerdo a su tipo de alimentación}

- Biodigestor continuo: Utilizado generalmente para saneamiento de aguas residuales o digestión de excretas animales. Se caracteriza por ser de gran capacidad (mayor a $6 \mathrm{~m}^{3}$ ), la materia de carga está altamente diluida en agua.

- Biodigestor semi-continuo: Utilizado para producción de biogás y fertilizantes, y para tratamiento de desechos. Puede ser cualquier capacidad, pero la primera vez se llena a un $80 \%$ de esta, dejando el $20 \%$ para introducir cargas pequeñas en intervalos regulares de uno o varios días. La producción de biogás es continua en el tiempo y alta la calidad de los fertilizantes producidos, debido a la renovación de la materia de carga.

- $\quad$ Biodigestor por lotes: Utilizado para tratamiento de desechos cuya concentración de solidos es muy alta (poca o nula disolución de agua).

Se carga una sola vez y se descarga cuando la producción de biogás decae considerablemente.

Dependiendo de la Estructura de
construcción tenemos las siguientes
posibilidades:


De planta con cúpula o campana flotante: el cual se compone de un digestor construido en mampostería o estructura de concreto y un depósito de gas móvil en forma de campana, la cual puede flotar directamente en la masa de fermentación o en un anillo de agua, dependiendo de la producción de biogás. La campana debe tener una guía que permita el movimiento vertical, cuya altura dependerá del volumen de gas almacenado. Es conocido también como biodigestor indio, y puede ser utilizado cuando se necesita de un abastecimiento continuo de biogás y fertilizante, caracterizándose por funcionar como depósito del gas producido, es decir, es el único tipo de biodigestores que tienen un depósito de biogás interior.

La planta con cúpula o campana fija: se compone de un digestor construido en mampostería y un domo fijo e inmóvil cerrado donde se almacena el biogás. Durante la producción de biogás, la masa de fermentación es desplazada hacia el tanque de compensación y cuando se extrae el gas, la masa líquida vuelve hacia el biodigestor. A través de constantes oscilaciones de la masa de fermentación en la parte superior de la cúpula se evita la formación de capa flotante. Es conocido también como biodigestor chino, y debido a que el gas debe ser liberado continuamente para reducir la presión interna, se utilizan en instalaciones donde el consumo sea continuo o para almacenar el biogás en un depósito externo

La planta balón o biodigestor tubular: La planta de balón se compone de un tubular en material plástico (polietileno, PVC, plastiloka, entre otros, y una combinación de éstos) completamente sellado, la entrada y la salida están sujetas directamente a las paredes de la planta. La parte inferior de la planta, en un $75 \%$ del volumen constituye la masa de fermentación, y en la parte superior, el $25 \%$ restante, se almacena el biogás. Este tipo de planta se recomienda para aquellos sitios donde predominan las temperaturas altas y constantes.

\section{Materiales orgánicos y sus diferencias}

Las actividades pecuarias y agrícolas producen materiales orgánicos que pueden ser tratados mediante el proceso anaeróbico. Otros materiales orgánicos de origen animal también pueden emplearse como sustrato para la obtención de biogás y bioabono. Los residuos vegetales, como paja, pasto y desechos de verdura pueden fermentarse anaeróbicamente debiendo previamente triturarse para evitar la formación de capa flotante y dar un tratamiento especial en plantas donde se hace una sola carga hasta que el material se descompone.

\section{Productos obtenidos de un Biodigestor}

Como se ha dicho anteriormente del proceso de un Biodigestor se obtendrán principalmente dos productos, el Biogas y el Biol, los cuales se describen a continuación.

\section{Biogás}

El biogás es una mezcla de gases producidos por fermentación anaeróbica. Tiene una alta concentración de metano (40\%-75\%), así como de vapor de agua (25\%55\%), que luego puede ser utilizado como combustible para la generación de energía calórica o eléctrica. Un biodigestor puede producir entre 1 y $2 \mathrm{~m}^{3}$ de biogás por día, lo que permite unas 2 horas de cocción de alimentos, aproximadamente.

Entre sus usos más difundidos, encontramos que provee energía para la iluminación de la vivienda y para la cocción de los alimentos. En relación con la iluminación de viviendas, esto es posible mediante el uso de lámparas a biogás, que consumen un promedio entre 0.12 y $0.15 \mathrm{~m}^{3}$ de biogás por hora, lo que equivale a una bombilla de $60 \mathrm{w}$, para su operación se usan mecheros similares al de una lámpara a kerosene.

En el caso de las cocinas, se requiere adaptar hornillas que permitan, la cocción de alimentos.

\section{Biol}

Biol es el efluente líquido que se obtiene de la biodigestión anaeróbica de materia orgánica, posee un poder fertilizante en los cultivos y los pastizales. 
El biol, puede tener diversas composiciones dependiendo el tipo de material orgánico que se degrade, es recomendable una debida caracterización de los componentes del biol a través de pruebas de laboratorio antes de utilizarlo como fertilizante, especialmente si se trata de cultivos para consumo humano.

\section{Variables que pueden monitorearse en un Biodigestor en la generación de Metano}

El objetivo principal de un biodigestor es la obtención del biogás, por lo que es necesario comprender cuales son los diferentes componentes químicos que se producen en la obtención del metano Tabla 1:

\begin{tabular}{|l|l|r|}
\hline \multicolumn{2}{|c}{ Componente } & $\begin{array}{r}\text { Composición } \\
\text { aproximada } \%\end{array}$ \\
\hline Metano & $\mathrm{CH}_{4}$ & $60-70$ \\
Gas Carbónico & $\mathrm{CO}_{2}$ & $30-40$ \\
Hidrógeno & $\mathrm{H}_{2}$ & 1.0 \\
Nitrógeno & $\mathrm{N}_{2}$ & 0.5 \\
Monóxido de Carbono & $\mathrm{CO}$ & 0.1 \\
Oxígeno & $\mathrm{O}_{2}$ & 0.1 \\
Ácido Sulfúrico & $\mathrm{H}_{2} \mathrm{~S}$ & 0.1 \\
\hline
\end{tabular}

Tabla 1 Componentes químicos del Biogás

El proceso de producción del biogás se desarrolla con la fermentación de la materia orgánica, en ello es indispensable considerar la temperatura a la que está sometida y el tiempo de reposo de la misma. De acuerdo a la temperatura y al tiempo al que está sometida la materia prima en el biodigestor, se puede clasificar la fermentación de la siguiente manera:

- $\quad$ Fermentación psicrofílica, para un rango de temperatura entre 10 y $20^{\circ} \mathrm{C}$ y más de 100 días de retención.

- $\quad$ Fermentación mesofílica, para un rango de temperatura entre 20 y $35^{\circ} \mathrm{C}$ y aproximadamente 30 a 40 días de retención.

- $\quad$ Fermentación termofilico, para un rango de temperatura entre 50 y $60^{\circ} \mathrm{C}$ y más de 8 días de retención. Este tipo de fermentación no es apropiada para plantas sencillas.

La materia prima al ser sometida a la fermentación libera en su mayor parte carbono (C) y nitrógeno $(\mathrm{N})$, lo que permite establecer la relación entre ellos $(\mathrm{C} / \mathrm{N})$, directamente relacionada a la producción de gas.
Una relación de 20:1 hasta 30:1 es aceptable, aunque el valor ideal es de 16:1, como un ejemplo de mezcla con materiales de fermentación de alto contenido en nitrógeno tenemos el estiércol de gallina y con material de fermentación con alto contenido de carbono el tamo de arroz, el cual generan una elevada producción de gas.

En la tabla 2. se puede apreciar la relación $\mathrm{C} / \mathrm{N}$ de diferentes productos:

\begin{tabular}{|l|r|}
\hline \multicolumn{1}{|c|}{ Sustancia } & $\begin{array}{r}\text { Relación } \\
\text { C/N }\end{array}$ \\
\hline Orina & 0.80 \\
Estiércol Equino & 25 \\
Estiércol Vacuno & 18 \\
Alfalfa & $16-20$ \\
Algas Marinas & 19 \\
Aserrín & 511 \\
Basura & 25 \\
Cáscaras de Papa & 25 \\
Paja seca de trigo & 87 \\
Paja seca de arroz & 67 \\
Tallo del maíz & 53 \\
Hojas secas & 41 \\
Estiércol de aves & 32 \\
Pasto & 27 \\
Estiércol Ovino & 29 \\
Estiércol de Cerdos & 13 \\
Excretas frescas humanas & 2.9 \\
\hline
\end{tabular}

Tabla 2 Relación C/N de varias sustancias

Niveles de amoniaco: Este parámetro es importante cuando se utilizan determinados materiales que contienen un alto porcentaje, como es el caso de los estiércoles de aves. Se recomienda que los niveles dentro de los digestores se mantengan por debajo de los 2000 $\mathrm{mg} / \mathrm{l}$, para lo cual se aumentan las diluciones de entrada del material. Nivel de acidez $(\mathrm{Ph})$ : Está en función de la concentración de $\mathrm{CO} 2$ en el gas, de la concentración de ácidos volátiles y de la propia alcalinidad de la materia prima. Las bacterias responsables del mecanismo de producción de biogás son altamente sensibles a cambios en el pH, oscilando entre 6 y 8 (es deseable un valor entre 7 y 7.2). El pH del lodo de fermentación indica si el proceso de fermentación transcurre sin problemas, y su medición indica el comportamiento de la carga de fermentación dentro del digestor para la producción de biogás.

\begin{tabular}{|l|l|}
\hline \multicolumn{1}{|c|}{ pH } & \multicolumn{1}{c|}{ Comportamiento } \\
\hline $7-7.2$ & Optimo \\
6.2 & Retarda la acidificación \\
7.6 & Retarda la amonización \\
\hline
\end{tabular}

Tabla 3 Niveles de PH de acuerdo a la concentración de $\mathrm{CO}_{2}$

ORTEGA-BUCIO, Lydia Gabriela, HERNÁNDEZ-HERNÁNDEZ, Fabiola, ZEA-PÉREZ, José Marcos y BECERRA-CHAVEZ, Adela. Biodigestor con variables monitoreadas. Revista de Energía Química y Física. 2019 


\begin{tabular}{|c|c|c|c|}
\hline Material Fresco & $\begin{array}{l}\% \\
\text { sólidos } \\
\text { totales }\end{array}$ & $\begin{array}{c}\% \\
\text { sólidos } \\
\text { orgánicos }\end{array}$ & $\begin{array}{l}\text { Litros de Biogás } \\
\text { por un Kg de } \\
\text { sólidos orgánicos }\end{array}$ \\
\hline Paja de arroz & 89 & 93 & 220 \\
\hline Paja de trigo & 82 & 94 & 250 \\
\hline Paja de maíz & 80 & 91 & 410 \\
\hline Hierba fresca & 24 & 89 & 410 \\
\hline Jacinto de agua & 7 & 75 & 325 \\
\hline Bagazo & 65 & 78 & 160 \\
\hline $\begin{array}{l}\text { Desecho } \\
\text { verduras }\end{array}$ & 12 & 86 & 350 \\
\hline
\end{tabular}

Tabla 4 Producción de Biogás con desechos vegetales

\section{Desarrollo del procedimiento}

Instrumentación para el monitoreo de variables en el Biodigestor.

\section{Sensor de Ph}

El sensor de $\mathrm{Ph}$ es nuevo en el mercado, pero ofrece las ventajas que cualquier programador busca que es un tiempo de respuesta corto y una señal análoga.

\section{Características}

- $\quad$ Rango de concentración detectable PHO14.

- $\quad$ Rango de temperatura $0-80{ }^{\circ} \mathrm{C}$.

- $\quad$ Tempo de respuesta menor a $5 \mathrm{~s}$.

- $\quad$ Voltaje de 5v.

- $\quad$ Corriente de trabajo de 5-10mA.

- Señal analógica de tensión

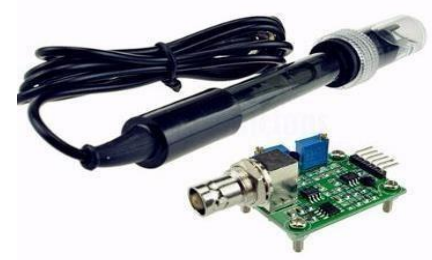

Figura 1 Sensor de $\mathrm{pH}$

\section{Sensor de Humedad}

El DHT22 Sensor digital de temperatura y humedad. Utiliza un sensor capacitivo de humedad y un termistor para medir el aire circundante, y muestra los datos mediante una señal digital en el pin de datos (no hay pines de entrada analógica). Es bastante simple de usar, pero requiere sincronización cuidadosa para tomar datos.
El único inconveniente de este sensor es que sólo se puede obtener nuevos datos una vez cada 2 segundos, así que las lecturas que se pueden realizar serán mínimas cada 2 segundos.

\section{Características}

- $\quad$ Alimentación: $3.3 \mathrm{Vdc} \leq \mathrm{Vcc} \leq 6 \mathrm{Vdc}$

- Rango de medición de temperatura: $40^{\circ} \mathrm{C}$ a $80{ }^{\circ} \mathrm{C}$

Precisión de medición de temperatura: $< \pm 0.5{ }^{\circ} \mathrm{C}$

Resolución Temperatura: $0.1^{\circ} \mathrm{C}$

- Rango de medición de humedad: De 0 a $100 \%$ RH

- $\quad$ Precisión de medición de humedad: $2 \%$ RH

- $\quad$ Resolución Humedad: $0.1 \%$ RH

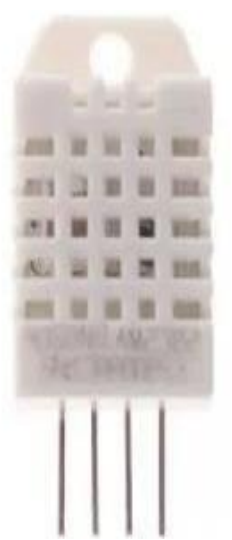

Figura 2 Sensor de Humedad

\section{Sensor de Temperatura}

El sensor de temperatura DS18B20 es un dispositivo que se comunica de forma digital. Cuenta con tres terminales: Vcc, GND y el pin Data. Este sensor utiliza comunicación por OneWire. Esté protocolo de comunicación permite enviar y recibir datos utilizando un solo cable. A diferencia de otros protocolos, que utilizan dos o más líneas de comunicación digital.

\section{Características}

- Sensor Digital

- $\quad$ Resolución de 9 y 12 bits

- $\quad$ Rango de operación de -50 a 125 grados

Centígrados

$-\quad$ Precisión +-0.5 grados
$-\quad$ Protocolo OneWire 


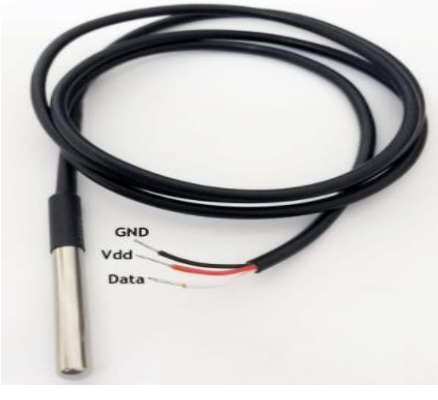

Figura 3 Sensor de Temperatura

\section{Sensor de gas MQ-2}

Especificaciones

Voltaje de operación:5V DC

Respuesta rápida y alta sensibilidad Rango de detección: 300 a 10000 ppm

Gas característico: 1000 ppm Isobutano

Resistencia de sensado: $1 \mathrm{k} \Omega 50 \mathrm{ppm}$ Tolueno a $20 \Omega$ kin

Tiempo de respuesta $\leq 30$ s

Temperatura de trabajo $-20{ }^{\circ} \mathrm{C}+55^{\circ} \mathrm{C}$

Humedad $\leq 95 \%$ RH

Contenido de oxígeno ambiental $21 \%$

Consume menos de $150 \mathrm{~mA}$ a $5 \mathrm{~V}$
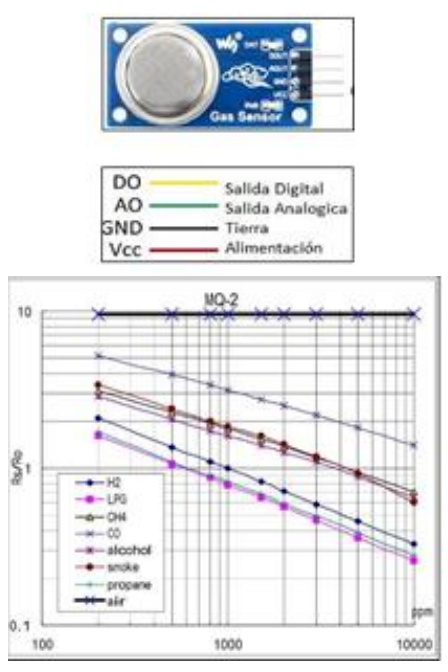

Figura 4 Sensor de gas y diagrama de conexión de entradas

En la figura 4. Se presenta la calibración del sensor para detectar metano en partículas por millón (ppm). En la gráfica se muestra una resistencia característica del sensor la sensibilidad en relación con el gas que detecte.

\section{Resultados}

\section{Etapa 1}

Sensor de Ph

Como podemos notar en la gráfica el valor de $\mathrm{Ph}$ ha oscilado entre los 5-6 aproximadamente con lo que se identifica que se necesita incrementar el nivel de $\mathrm{Ph}$ para que se encuentre entre 6,5 y 7 , es decir un nivel de acidez neutro. Para el sensor de $\mathrm{Ph}$ se obtuvieron las siguientes mediciones y de ahí se generó el grafico:

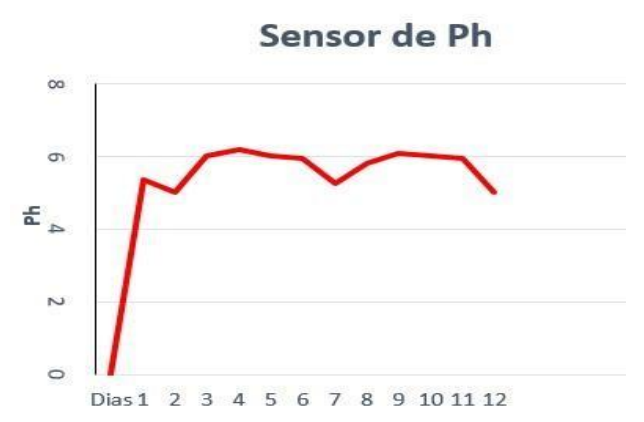

Gráfica 1 Sensor $\mathrm{Ph}$

\section{Sensor de Temperatura}

Las lecturas del sensor de Temperatura se muestran en la gráfica. Se observa que el Biodigestor se encuentra en la etapa psicrofílica de un Biodigestor que es la etapa de las primeras 2 semanas en la que la temperatura se encuentra por debajo de $\operatorname{los} 25^{\circ} \mathrm{C}$.

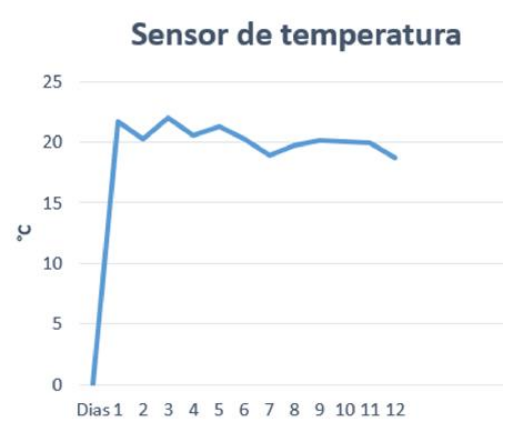

Gráfica 2 Sensor de Temperatura

\section{Sensor de Humedad}

En la gráfica se identifica que el porcentaje de humedad es importante pues con este podemos hacer que el proceso de degradación se agilice y sea más factible. 


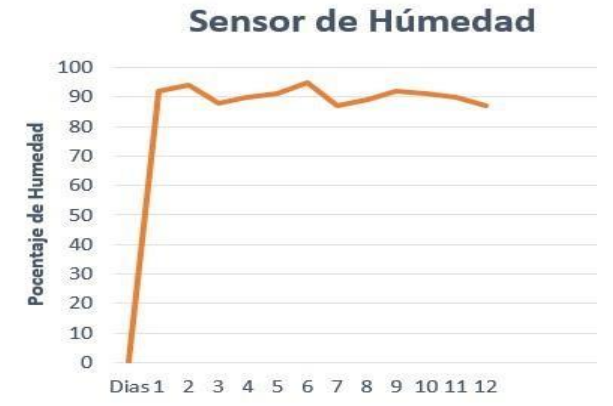

Gráfica 3 Sensor de humedad

\section{Sensor de gas MQ2}

Parte de las pruebas fue la detección de Gas metano con el Sensor MQ2 en ppm y los resultados son satisfactorios debido a que nuestro sensor marca la presencia de gas metano, gas que sale del bidón hacia la cámara de almacenamiento.Para el sensor MQ2 se obtuvieron los siguientes resultados;

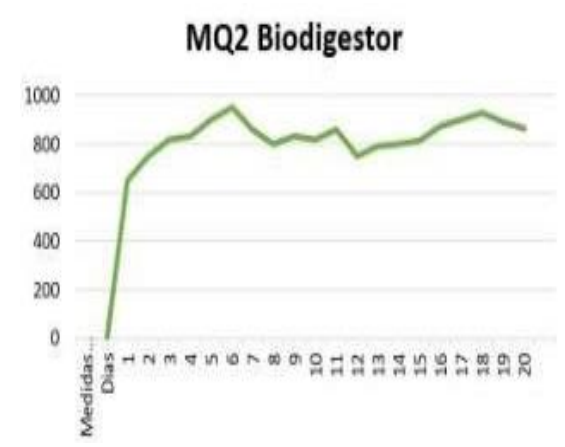

Gráfica 4 Sensor de Gas

\section{Etapa 2}

Se realizaron pruebas con los componentes existentes y los que estaban de reserva para comprobar su funcionamiento. Dentro de la información proporcionada no se encontraron datos sobre algunos componentes que tuvieron que ser investigados, tales como características de funcionamiento de algunos sensores (rango) algunos datos relevantes fueron que el sensor de PH no puede estar realizando pruebas continuamente por lo que este sensor no podría estar dentro del biodigestor realizando pruebas constantes como se tenía pensado en el diseño anterior, por lo que este y la programación anterior se cambiaron.
Luego se procedió a revisar las programaciones que se hicieron para el biodigestor, estas consistían en dos programas, el primero consistía en el funcionamiento del sensor de temperatura junto con el sensor de PH, además de la programación de una pantalla LCD en el cual estos datos serian mostrados a la vez que en le puerto serial; el segundo consistía en el funcionamiento de la sensor de gas y el de humedad, también con una pantalla LCD para mostrar los datos obtenidos con los sensores y mostrarlos también por el puerto serial.

De estos 2 programas el único que presento un problema de funcionamiento fue el primero en la parte de la obtención de datos del sensor de $\mathrm{PH}$, por lo que se buscó generar una programación para su correcto funcionamiento además de modificar la parte que hacia el continuo uso de este mismo, a lo que se decidió agregar un botón el cual cuando se mantuviera presionado accionaria el funcionamiento del sensor de PH y mostraría los valores, dejando el sensor de temperatura tal y como estaba.

Tras realizar tales cambios se procedió a probar los sensores junto con la programación para obtener series de valores como muestra del buen funcionamiento.

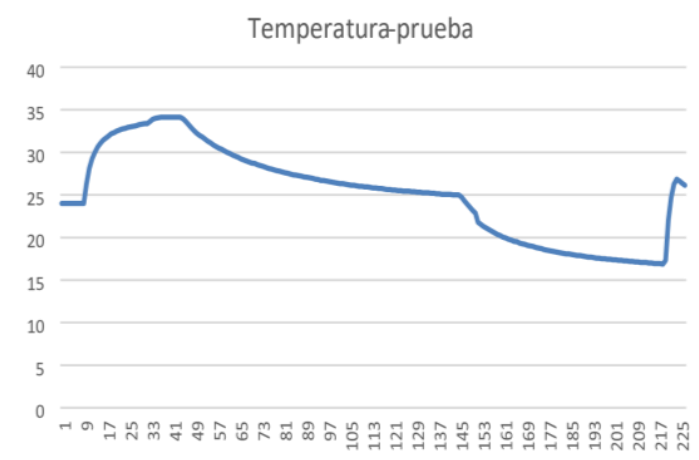

Gráfica 5 Prueba del sensor de Temperatura

En la gráfica una se muestran los datos obtenidos del sensor de temperatura mediante la comunicación serial, donde se tomaron 255 muestras, las primeras 15 son la temperatura ambiente, de allí a la muestra 45 se le aproximó a una fuente de calor, de la muestra 46 a la 150 se le retiró la fuente de calor por lo que desciende el registro de temperatura para llegar a la temperatura ambiente, de la muestra 151 a la 220 se le expuso a una temperatura baja por contacto, y por último de la 221 a la 225 se acercó de nuevo una fuente de calor. 


\section{Sensor de PH}

En la siguiente tabla se enlistan datos obtenidos con el Biol generado en la prueba.

\begin{tabular}{|c|}
\hline Sensor PH \\
\hline Ph:6.04 \\
Ph:6.04 \\
Ph:6.04 \\
Ph:6.04 \\
Ph:5.98 \\
Ph:6.04 \\
Ph:5.98 \\
Ph:5.98 \\
Ph:5.98 \\
Ph:6.04 \\
Ph:6.04 \\
Ph:6.04 \\
Ph:6.04 \\
Ph:6.04
\end{tabular}

Tabla 5 Sensor PH pruebas

\section{Sensor de Humedad}

En la Gráfica 6. se observan los datos obtenidos del sensor de humedad, durante la cual al igual que para la prueba de temperatura le fue expuesto a cambios como meterlo y sacarlo de un bidón con agua, donde las partes altas de la gráfica marcan el momento en que fue introducido dentro, y las otras estando fuera del bidón.

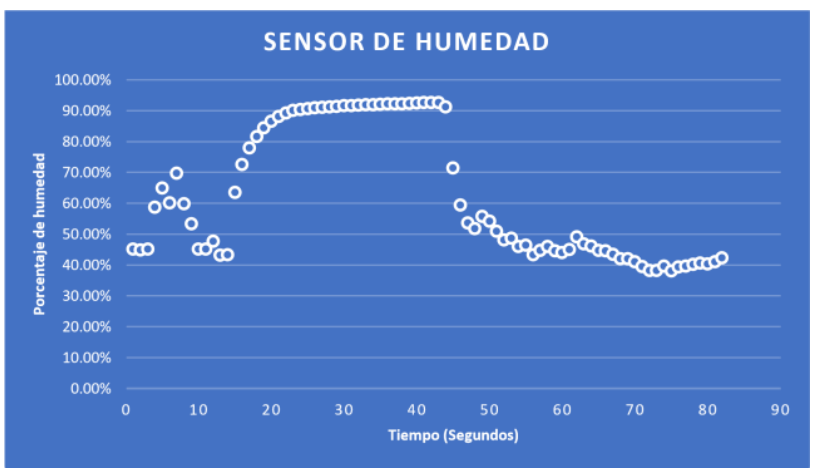

Gráfica 6 Sensor de Humedad pruebas

\section{Sensor de Gas}

En la gráfica obtenida con el sensor de gas tomadas durante la prueba del sensor de humedad, donde el sensor fue expuesto a gas butano de un encendedor cuando se marcan puntos arriba del cero; solo falta aclarar que a pesar de no cambiar el encendedor en la prueba los datos no llegan a tener un patrón o valor definido, esto se debe a que lo que mide son partículas por millón, por lo que factores como el aire afectan mucho sus medidas.

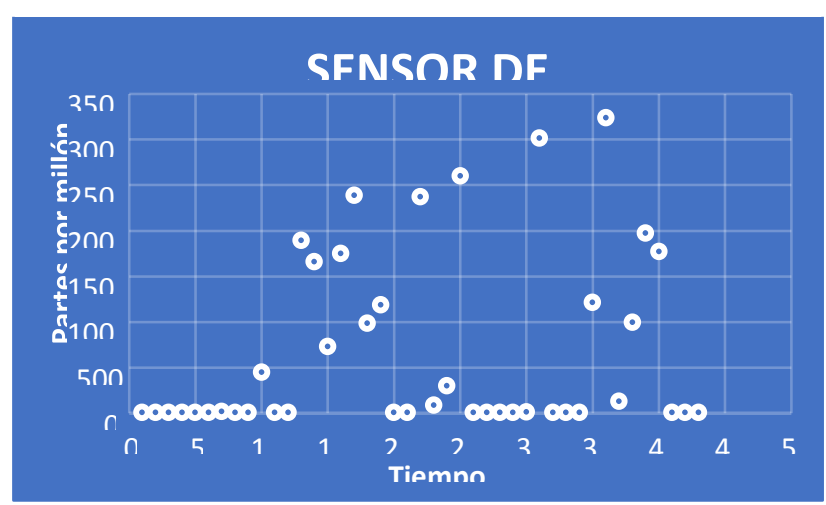

Gráfica 7 Sensor de gas, pruebas

\section{Prototipo del Biodigestor}

Después de comprobar la funcionalidad de los sensores y el programa se procedió a construir el prototipo físico el cual se realizaría sobre un bidón, colocándole una llave de paso cercana al fondo, introduciendo el sensor de humedad junto con el de gas en la tapa del bidón, además de hacer una pequeña perforación en la cual se introduciría el sensor de temperatura, y todos los demás componentes Figura 5. y Figura 6.

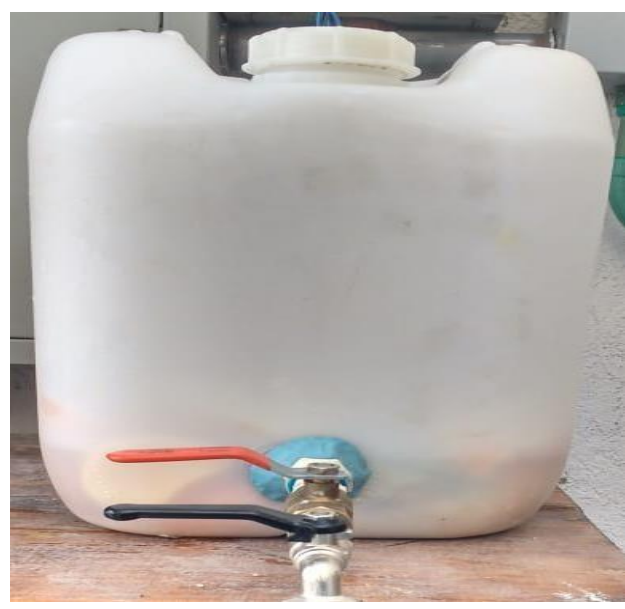

Figura 5 Biodigestor Prototipo
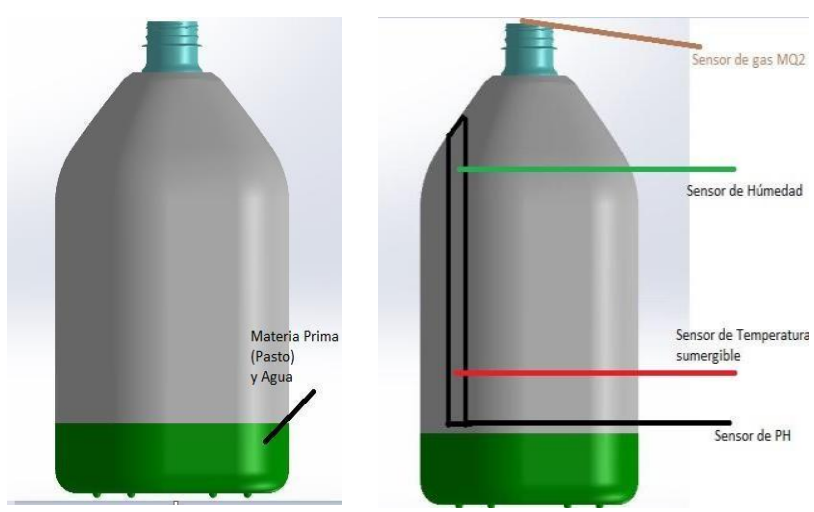

Figura 6 Sensores dentro del Biodigestor

ORTEGA-BUCIO, Lydia Gabriela, HERNÁNDEZ-HERNÁNDEZ Fabiola, ZEA-PÉREZ, José Marcos y BECERRA-CHAVEZ, Adela. Biodigestor con variables monitoreadas. Revista de Energía Química y Física. 2019 
Los Arduino estarán colocados sobre el bidón, se colocó un pequeño circuito en el cual a través de un eliminador (cargador de celular) se podía alimentar dos Arduinos para depender de un alimentación de $127 \mathrm{~V}$ de cualquier enchufe en vez de una fuente o computadora que suministre los $5 \mathrm{~V}$ necesarios.

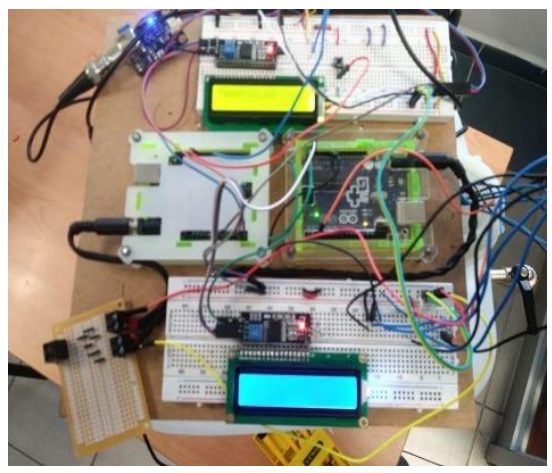

Figura 7 Arduinos para la programación de los Sensores del Biodigestor

Implementación de Pantallas LCD por medio de comunicación I2C. Utilizando 2 pantallas LCD, se pueden visualizar los datos generados por los sensores de una manera más sencilla.
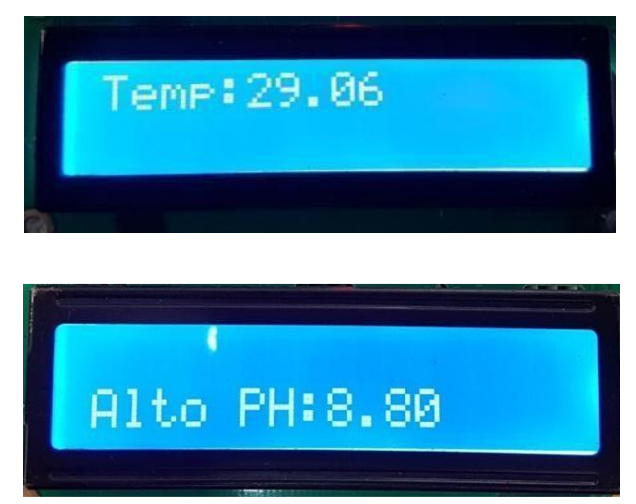

Figura 8 Pantallas LCD permitiendo la visualización de los valores obtenidos en los sensores

Se realizó una placa especial para la obtención de datos y conexión a los sensores de gas y humedad, con la misma se obtuvieron los últimos datos generados por la muestra.

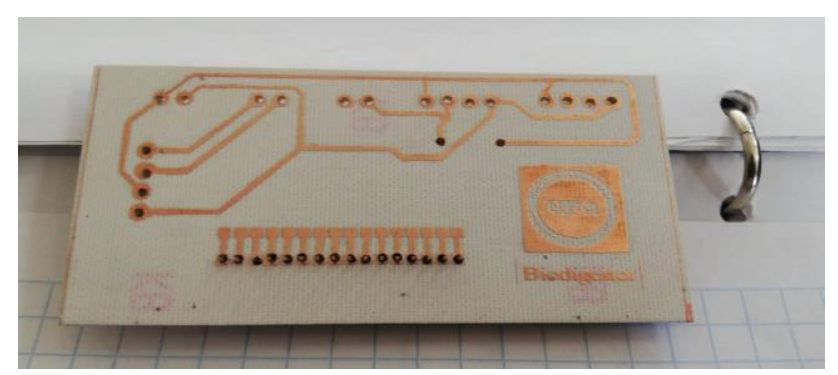

Figura 9 Placa de obtención de datos
Las conexiones de entrada y salida de los sensores, se realiza por la parte superior del bidón como se muestra en la figura 10.

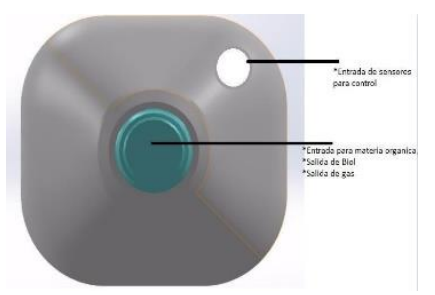

Figura 10 Posición de entrada y salida de los cables en el prototipo del Biodigestor

\section{Diseño del Prototipo Final}

El prototipo final se encuentra conformado por 3 biodigestores, uno de ellos será el único controlado con este aseguramos que el sistema se encuentra trabajando en condiciones óptimas. Las salidas de los biodigestores se encontrarán conectadas a un botellón con agua en el que se encuentra nuestro sensor MQ2 para captar cuantas ppm generan nuestro biodigestores y también contiene agua que nos funciona para filtrar el gas y para notar a simple vista que se está generando gas. En el tercer biodigestor tendrá el acoplamiento a un tanque de gas para contener la producción de metano, este podrá ser conectado a una parrilla especial para metano en donde se podrá realizar la cocción o calentamiento de alimentos.

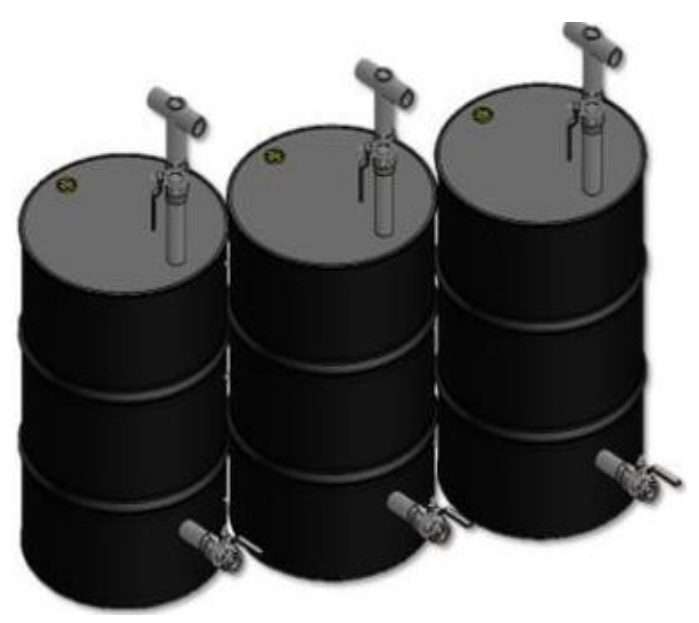

Figura 11 Sistema de tres Biodigestores 


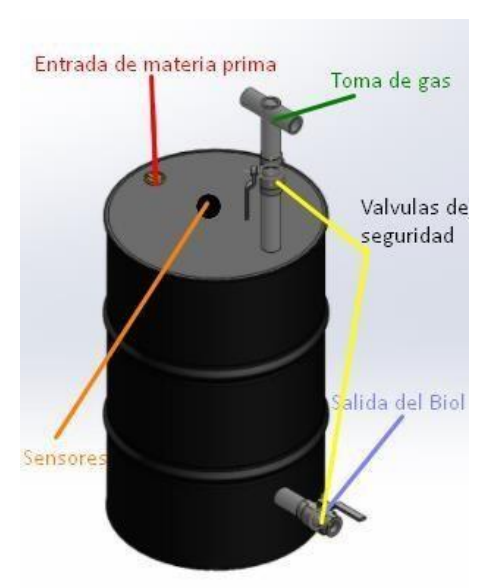

Figura 12 Entradas y salidas del Biodigestor controlado en el Diseño definitivo

\section{Agradecimiento}

Se agradece a la Universidad Politécnica de Querétaro el apoyo proporcionado para realizar este proyecto y lograr diversos apoyos económicos como es la convocatoria para Nuevos Talentos.

Un reconocimiento particular a cada uno de los estudiantes que conformaron los equipos en las diferentes etapas de este proyecto.

\section{Conclusiones}

Los Biodigestores controlados son una propuesta de alternativa práctica, innovadora y funcionalmente satisfactoria, el biogás es una fuente energética de bajo costo para cocinar o calentar lo que suple las necesidades básicas del hogar, como se sabe todo el proceso para la generación del mismo no requiere de procesos industriales o químicos lo que hace que este producto sea amigable con el medio ambiente siendo una alternativa sustentable a los recursos no renovables como lo es el gas tradicional. Por otra parte, no aumenta la cantidad de dióxido de carbono en la atmosfera y principalmente permite reducir la basura que generamos a diario $\mathrm{y}$ aprovechar los residuos para generar combustible.

Como se sabe hay varias zonas rurales en los diferentes países que no cuentan con redes de gas y el acceso a tanques de gas no solo es costoso, sino que en muchos casos se hablan de comunidades de escasos recursos lo cual hace imposible para varias familias el adquirir uno.
Para ello el Biogás es una alternativa de fuente energética que logra satisfacer las necesidades básicas de las familias y hablando de manera empresarial para las diferentes PYMES. Durante los últimos años es que se ha escuchado hablar acerca de los biodigestores, pero la importancia de un Biodigestor controlado es grande pues da un cambio radical a lo que actualmente se conoce, ya que permite saber si alguna de las variables controladas se encuentra fuera del nivel óptimo para un mejor proceso de descomposición, también se obtiene mayor seguridad y se conoce la calidad del gas que se está generando.

El uso de sensores para el control es lo que diferencia este proyecto, mientras el biodigestor se encuentre en sus rangos óptimos la producción del metano será continua y de gran calidad.

El impacto que tiene un biodigestor controlado actualmente es de gran importancia no solo para la parte tecnológica sino para lo social, ambiental y económico, se cree que aún hay mucho por mejorar y trabajar pues se puede llegar a un modelo de mayor calidad y control.

El proyecto Biodigestor con variables monitoreadas logró obtener Biogás a partir de la materia orgánica de la universidad, el monitoreo de los sensores fue registrado en una etapa de prueba para el control de mismo.

La validación del funcionamiento de sensores proporciona datos importantes para continuar el desarrollo del proyecto. Se encuentra una problemática fundamental en la hermeticidad del sistema en dos partes, la generación del gas, lo cual todavía no se puede asegurar y el olor generado por la materia orgánica.

También es importante verificar el funcionamiento de los sensores en operación prolongada para comprobar si el diseño actual es eficaz para el prototipo final.

\section{Referencias}

[1] JIMÉNEZ VALDEZ, Mario Iván. (2011). Introducción. En sistema Biodigestor Controlado (3). DF: Instituto Politécnico Nacional. 
[2] OLAYA ARBOLEDA, Yeison. (2009). Fundamentos para el diseño de un biodigestor. PALMIRA: Universidad Nacional De Colombia Sede Palmira.

[3] GONZÁLEZ, Ocatvio.(2010). Biodigestores. Universidad Nacional de Perú.

[4] CORONA ZÚÑIGA, Iván. (2007). Biodigestor. Hidalgo: Universidad Autónoma del Estado de Hidalgo.

[5] SOUZA, Josué, SCHAEFFER, Lirio. (2013). sistema de Compresión de Biogás y Biometano. Brasil: Universidad Federal de Rio Grande do Sul. 